\title{
Restoring anterior tooth aesthetics with a Non- Rigid connector - A modified approach (Case Report)
}

\author{
Dr.Shruti Gill ${ }^{1}$, Dr.Mudita Chaturvedi ${ }^{2}$, Dr. Saurabh Chaturvedi ${ }^{3}$, \\ Dr.Rahul Srivastava ${ }^{4}$
}

1- Senior Lecturer, Department of Prosthodontics, Terna dental college, Mumbai Mharashtra, India

2- Senior Lecturer, Department of Oral and Maxillofacial Pathology, Career Post graduate institute of dental sciences \& Hospital, Lucknow, Uttar Prades,India

3- Senior Lecturer, Department of Prosthodontics, Career Post graduate institute of dental sciences \& Hospital, Lucknow, Uttar Prades, India

4- Senior Lecturer, Department of Prosthodontics, Career Post graduate institute of dental sciences \& Hospital, Lucknow, Uttar Pradesh, India

\begin{abstract}
Aesthetic anterior tooth restoration is one of the greatest challenges for dentists in clinical practice. Patients expect contemporary dentistry to provide restorations that reproduce natural beauty while ensuring stability and functionality. The Fixed dental prosthesis fabrication requires equal consideration to be given to the retainer, pontic and connector design.

The excessive flexing of the long-span fixed dental prosthesis, which varies with the cube of the length of span, can lead to material failure of prosthesis or to an unfavorable response. The size, shape, and position of the connector leads to the success of prostheses, as it prevent the distortion and fracture of the prosthesis. The conventional use of a non rigid connector aids in compensating for the difference in the resistance and retention form between the abutments. This paper describes a modified technique of restoring anterior aesthetics with non rigid connector.
\end{abstract}

Keywords - Fixed Dental Prosthesis, Non Rigid Connectors, Pier Abutment.

\section{Introduction}

Aesthetic anterior tooth restoration is greatest challenge in today's clinical practice. Patients expect contemporary dentistry to provide restorations that reproduce natural beauty while ensuring stability and functionality. In achieving this goal, the dentist faces numerous dilemmas. Tooth shape and size are both subordinated to the anatomical peculiarities of the jaws, face, lips, and nose. However, utilizing appropriate material and technique, excellent aesthetics can be achieved with restoration of optimum function.

The occlusal forces applied to a fixed dental prosthesis (FDP) are transmitted to the supporting structures through the pontic, connectors, and retainers. Variables that may influence the longevity of an FDP and its abutment include occlusion, span length, bone loss, and quality of periodontium ${ }^{1}$. The excessive flexing of the long-span FDP, which varies with the cube of the length of span, can lead to material failure of prosthesis. Stress concentration around the connectors of the prosthesis and in the cervical dentin area near the edentulous ridge plays an important role in the potential for failure in long-span $\mathrm{FDP}^{2}$. The conventional use of a non-rigid connector (NRC) aids in compensating for the difference in the resistance and retention form between the abutments. The design and passive fit of NRC is critical to the success of a long-span FDP.

The connector that permits limited movement between the otherwise, independent members of the FDPs is the NRC .The NRC could be made by an incorporation of prefabricated wax patterns, prefabricated inserts or by custom-milling machine ${ }^{3}$. The connector should provide adequate access for the maintenance of oral hygiene and also should avoid display of metal. The connector should be elliptical with a definite curve facio-lingually and should have a smooth transition Mesio-distally ${ }^{4}$.

It should be prepared within the contours of the retainers with the male component (Tenon) attached to the pontic ${ }^{5}$. The NRCs have certain limitations such as it cannot be given in abutments having a large pulp size and reduced clinical crown height.

\section{Case Report}

A 23 year old female patient with missing upper anterior teeth since 7 years due to trauma reported to the department. Oral examination revealed missing 11121322 23. [Fig1] . The only teeth in upper anterior segment was 21 which was Supraerupted with adequate bone support radiographically. This tooth was considered to serve as a pier abutment. The lower anteriors also showed marked supraeruption which were almost touching the upper residual ridge. After thorough examination diagnostic impressions were made with 
irrereversible hydrocolloid, diagnostic cast were poured, facebow record was made and transferred on articulator.

Different treatment options were evaluated like planned partial denture, implant prosthesis, Fixed dental prosthesis. As the patient was young she refused to have planned partial denture and could not afford implant prosthesis. Hence FDP utilizing 21 as pier abutment with NRC was considered to be an optimal treatment plan. The probable outcome of treatment was discussed with the patient. For convenience the treatment was planned in two phases.

\section{Phase I - Correction of the lower anterior occlusal plane.}

In this case the occlusal scheme was group function and had protrusive interferences. These interferences were evaluated with the articulating paper $40 \mu$ thick (Bausch) in patients mouth and on the articulator and corrected with selective grinding before the start of the treatment. As the lower anterior teeth were supraerupted it required intentional root canal treatment for the correction of occlusal plane. Intentional RCT with 313233414243 and 21 was planned. Mock preparation was done and a diagnostic wax up was completed where the anterior guidance for the patient was established [Fig2]. Tooth preparation was done with

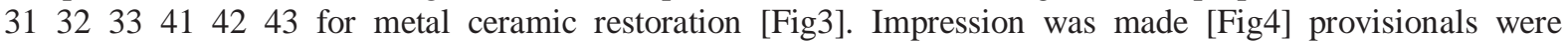
cemented and individual metal ceramic crowns were fabricated and cemented [Fig5].

\section{Phase II - Correction of maxillary arch}

In Maxillary arch, tooth preparation with 1415212425 was done [Fig6]. Gingival tissue retracted and impression was made with putty reline technique for the preparation of working model [Fig7]. Provisionals were made and cemented with temporary luting cement. Fixed dental prosthesis with NRC was fabricated in wax with a keyway (Mortise) on the mesial and distal aspect of 21 and key (Tenon) on 11 and 22 and then cast [Fig8]. After metal try in ceramic built up was done and again tried, necessary adjustments were made and finally glazed [Fig9]. 21 was cemented first and then other two bridges were tried for their fit and cemented [Fig10,11,12].

\section{Discussion}

In this case Pier abutment with 21 was present which can promote a fulcrum-like-situation that can cause the weakest of the terminal abutments to fail and cause intrusion of the pier abutment. The connector design was altered in 21 region where maximum tension can occur, thus the characteristic stress pattern was optimized to improve the survival time of three-unit FDPs. W. Oh, N. Götzen and K.J. Anusavice ${ }^{6}$ in their study suggest that stresses are better distributed with broadly curved connectors than through the use of more sharply curved connector geometries. PV Badwaik, AJ Pakhan ${ }^{7}$ described the use of NRCs in posterior segment.

This case has described the use of NRC in the anterior aesthetic zone with modification. A balancing phenomena was incorporated with Tenon on mesial and distal side of 21 . As the teeth were misaligned the preparation for FDP got complicated and it was avoided by NRC. The component parts did not have a common path of placement and therefore segmenting of long span FDPs with shorter components resulted in compensating the difference in resistance and retention form between the abutments.

The non-rigid interconnection employed a semi-precision type of attachment. Hence, the possible problem of overloading the tooth or potential intrusion phenomena that might be associated with tooth migration was not noticed with NRCs. This case was followed up for $1 \mathrm{yr}$ and the radiographs showed no bone loss [Fig 15]. Thus it demonstrated that stunning cosmetic result could be achieved with appropriate diagnosis, treatment planning, along with an adequate knowledge of the techniques available.

\section{FIGURES}

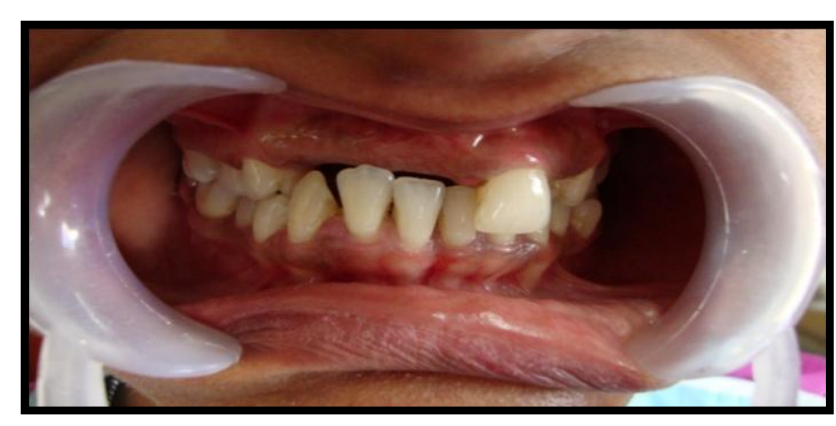

Fig.1 Intraoral frontal view 


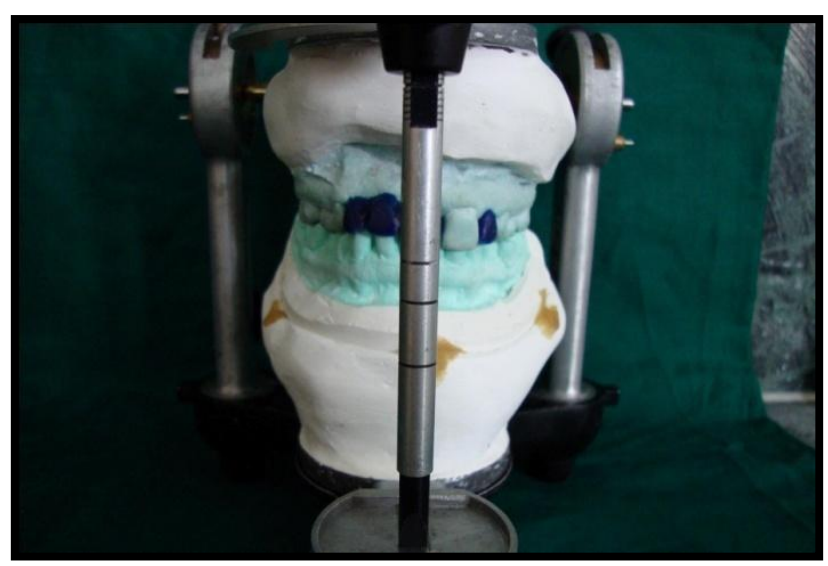

Fig 2 Diagnostic Mounting and wax- up

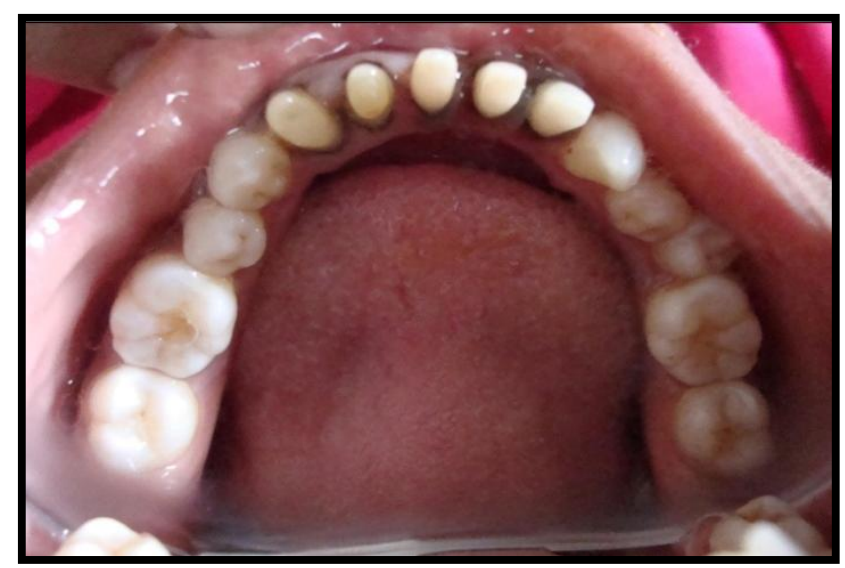

Fig 3 Tooth preparation with 313233414243

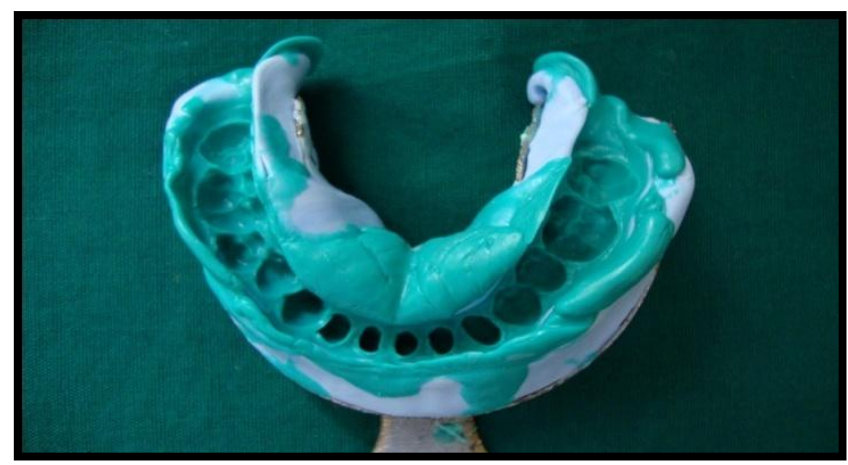

Fig.4 Mandibular Final Impression

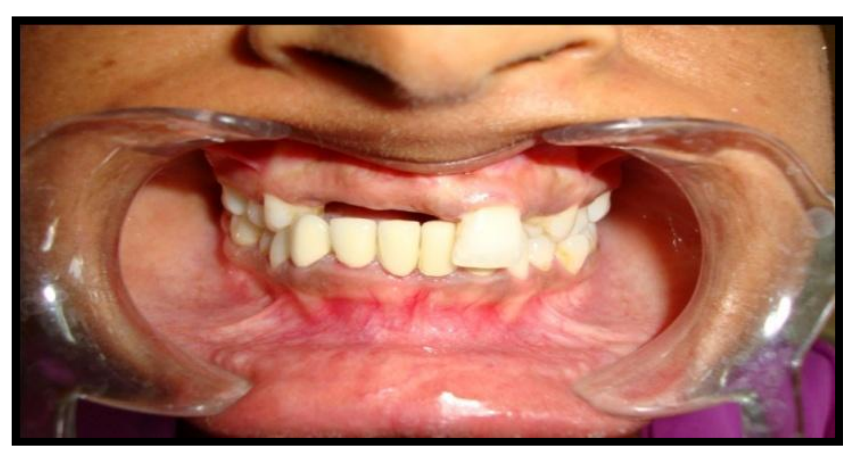

Fig 5 Corrected mandibular occlusal plane 


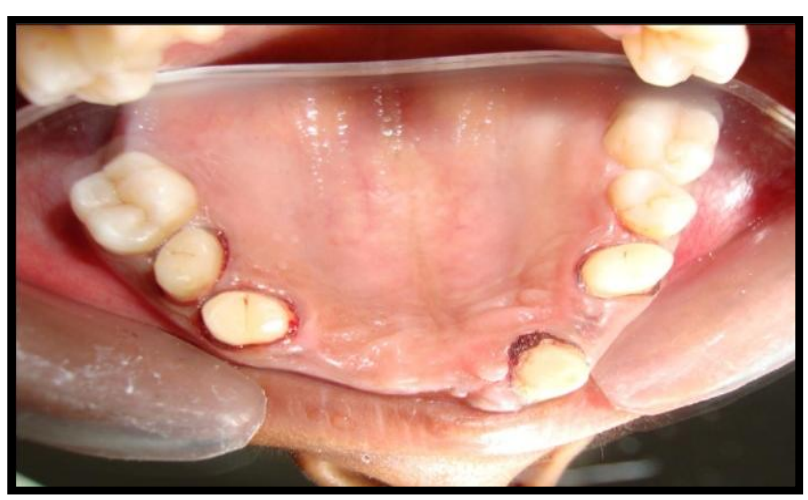

Fig 6 Tooth preparation with 1415212425

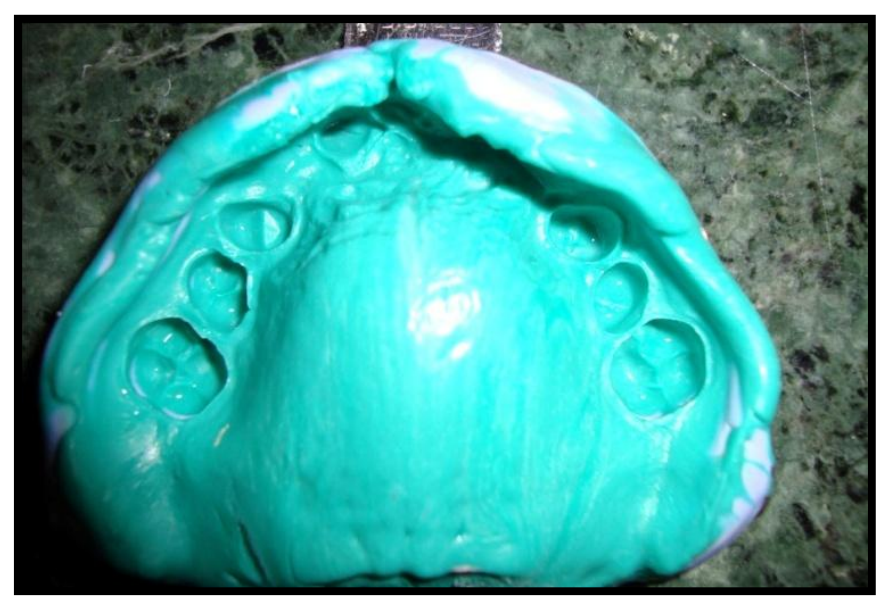

Fig 7 Maxillary Final Impression

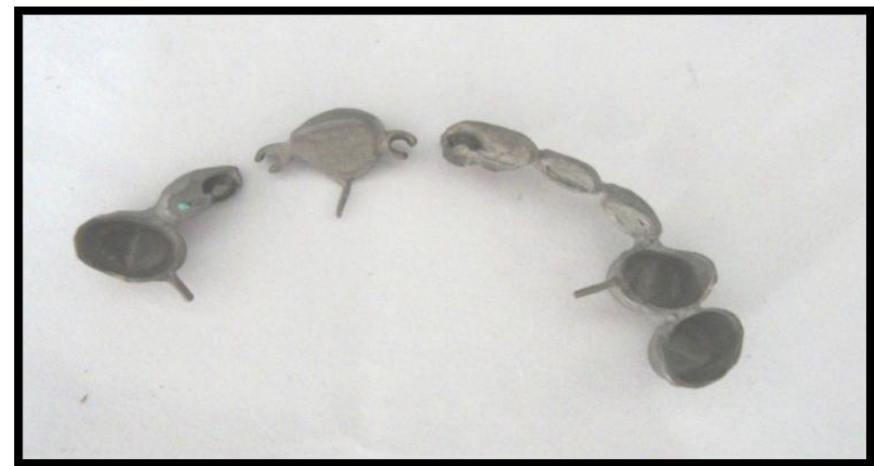

Fig 8 NRC on mesial and distal of 21

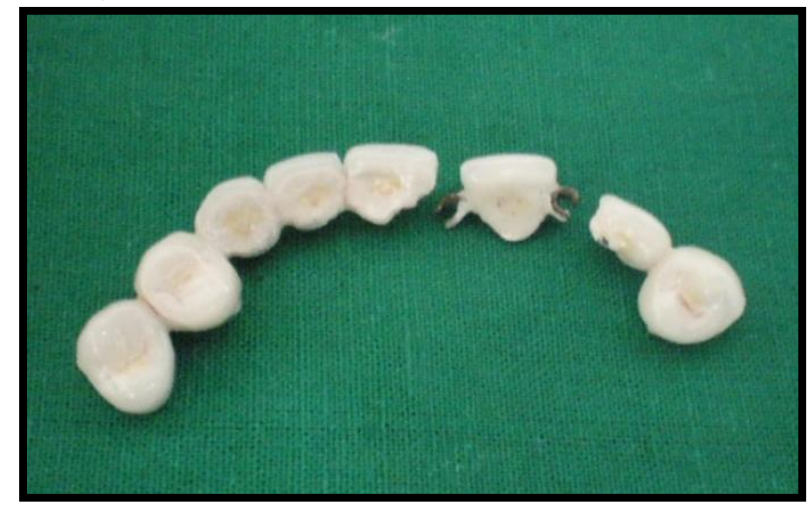

Fig 9 Glazed Prosthesis 


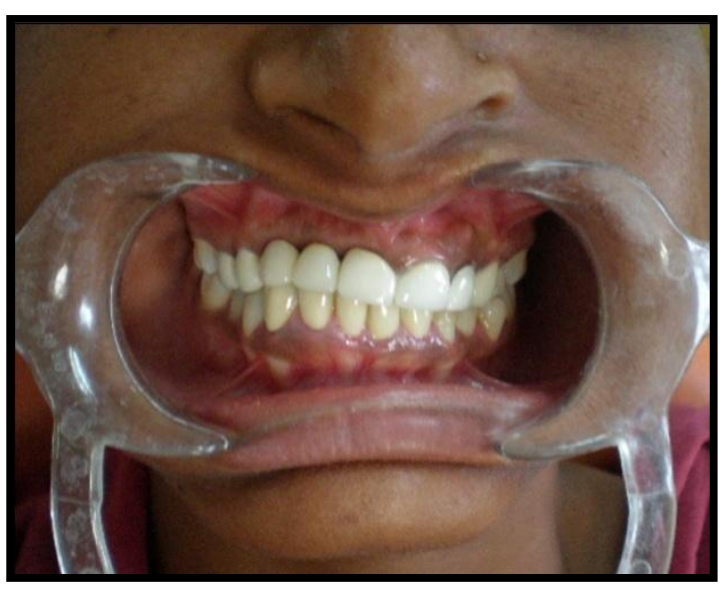

Fig 10 Cemented Prosthesis

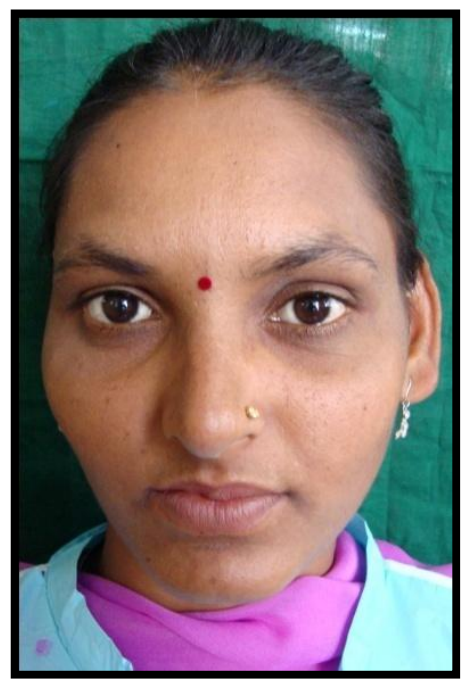

Fig 11 Preoperative Frontal View

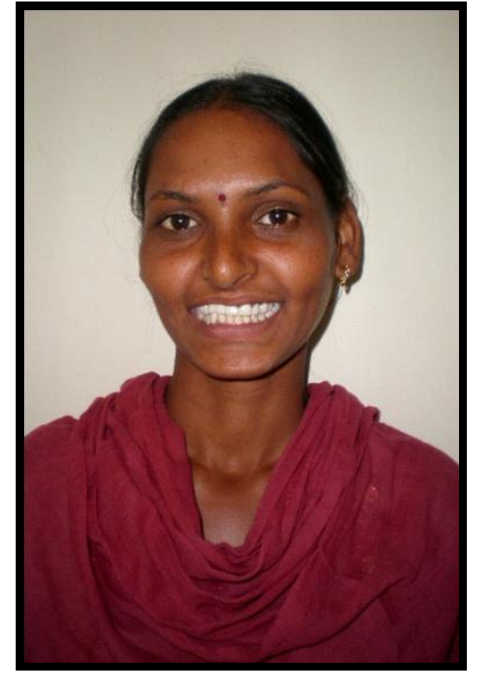

Fig 12 Postoperative Frontal View

\section{CONCLUSION}

Non-rigid interconnection between pontic and natural teeth to support fixed dental prosthesis has been used. It has been recommended that the use of this type of internal interconnection might allow retrievability of the prosthesis as well as reducing overloading of the abutment teeth ${ }^{8}$. Thus the design and passive fit of NRC is critical to the success of a long-span FDP.

\section{REFERENCES}

[1]. Rosenstiel S, Land M, Fujimoto J. Contemporary fixed prosthodontics. 3rd Edn. Harcourt: India; 2002. p. $65-81$.

[2]. Shillinburg HT Jr, Hobo S, Whitsett LD, Jacobi R, Brackett SE. Fundamentals of fixed prosthodontics. 3rd edn. Passi; India; 1997. p. 95-100.

[3]. Sherring-Lucas, Martin. Attachments for prosthetic dentistry: Introduction \& application. Quintessence: London; 1994.

[4]. Pissiotis \& Michalakis - An Esthetic \& Hygienic Approach To The Use Of Intracoronal Attachments As Interlocks In Fixed Prosthodontics. J Prosthet Dent 1998;79:347.

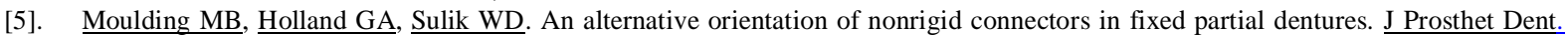
1992 Aug;68,2:236-8.

[6]. W. Oh, N. Götzen and K.J. Anusavice Influence of Connector Design on Fracture Probability of Ceramic Fixed-partial Dentures Journal of Dental Research 2002;81,9:623-627.

[7]. PV Badwaik, AJ Pakhan Non-rigid connectors in fixed prosthodontics: Current concepts with a case report. Journal of Indian Prosthodontic Society 2005; 5,2:99-102.

[8]. Bader H. Al-Ansari, Use of non-rigid connection between natural teeth and implants to support fixed partial denture. Two years clinical evaluation. The Saudi Dental Journal, 1996;8 2:96-99. 